\title{
Monvillea phatnosperma (CACtaceae), nueVa cita PARA La Flora Argentina
}

\author{
LUIS J. OAKLEY1 , VIRGINIA Y. MOGNI ${ }^{1}$ y DARIÉN E. PRADO ${ }^{1}$
}

\begin{abstract}
Summary: Monvillea phatnosperma (Cactaceae), a new record for the Argentinian Flora. The species Monvillea phatnosperma (K. Schum.) Britton \& Rose (Cactaceae), is cited and collected for the first time for Argentina, in the province of Formosa. Its distribution is extended within the Great Chaco expanse, since it was only known from Paraguay until now. Some aspects of its morphology, taxonomy and nomenclature are considered.
\end{abstract}

Key words: Monvillea phatnosperma, Cactaceae, Argentina, Chaco.

Resumen: Se cita por primera vez para la Argentina a Monvillea phatnosperma (K. Schum.) Britton \& Rose (Cactaceae), coleccionada en la provincia de Formosa. Se extiende además su área de distribución dentro del 'Gran Chaco', dado que sólo estaba registrada para Paraguay. Se realizan algunas consideraciones morfológicas, taxonómicas y nomenclaturales sobre la misma.

Palabras clave: Monvillea phatnosperma, Cactaceae, Argentina, Chaco.

\section{INTRODUCCIÓN}

El género Monvillea fue creado por Britton $\&$ Rose (1920) para un grupo de especies sudamericanas de hábito arbustivo o subarbustivo, anteriormente incluidas en Cereus. Se caracterizan por su hábito postrado a semi-erecto (raro arbóreo), tallos cilíndricos, relativamente delgados, con costillas bajas y obtusas; flores mayores a $7 \mathrm{~cm} \mathrm{de}$ longitud, efímeras, nocturnas, con pocas escamas sobre el pericarpelo, inermes o esporádicamente con alguna espina pequeña; frutos ovoides a piriformes, dehiscentes por una rajadura longitudinal, con semillas lisas, relativamente pequeñas, brillantes. El género presenta una distribución discontinua a través del centro-este de Sudamérica (Brasil, este de Bolivia, Paraguay y Argentina), y luego en Ecuador y norte de Perú.

${ }^{1}$ Cátedra de Botánica, Facultad de Ciencias Agrarias, UNR. Campo Villarino, S2125ZAA Zavalla, Prov. Santa Fe, Argentina. E-mail: loakley@unr.edu.ar
Para la Argentina se citaron cuatro especies: Monvillea cavendishii (Monv.) Britton \& Rose, M. euchlora (K. Schum.) Backeb., M. krapovickiana R. Kiesling y M. spegazzinii (F.A.C. Weber) Britton \& Rose (Kiesling et al. 2008, Kiesling, 2010). En el presente trabajo se cita por primera vez para la flora argentina la presencia de Monvillea phatnosperma (K. Schum.) Britton \& Rose, encontrada y registrada en el centro-oeste de la provincia de Formosa.

\section{Material y Método}

El material utilizado para el registro de esta especie fue coleccionado en un viaje de campaña a las provincias de Chaco, Corrientes y Formosa, durante el mes de marzo de 2011, en el marco del Proyecto: "Especies y comunidades vegetales del Dominio Chaqueño Húmedo: ecología y taxonomía (SECYTUNR)". El mismo fue depositado en el herbario UNR. Para la identificación del material se consultó bibliografía específica sobre la familia Cactaceae (Britton \& Rose, 1920; Kiesling 1984 y 1994). 
Bol. Soc. Argent. Bot. 48 (3-4) 2013

\section{Resultados}

Monvillea phatnosperma (K. Schum.) Britton \& Rose, The Cactaceae 2: 24, 1920.

=Cereus phatnospermus K. Schum., Monat. kakteenk. 9: 186, 1899. Typus: Paraguay, Porongo, Anisit 27, 18/II/1888 (Holotypus: B, foto del tipo!).

Arbusto muy ramificado, ramas suculentas decumbentes o ascendentes, de 1-2 m de largo o más, de color verde-oscuro. Ápice obtuso, hemisférico, no tuberculado; costillas 4-5, redondeadas, obtusas, bien definidas hasta el ápice. Areolas ca. 0,8 mm diám., separadas 3-4 cm, las jóvenes con indumento blanquecino notable. Espinas rectas, subuladas, marrones; radiales 5-6, desiguales, de 0,5-2,5 cm; central 1 , de $2,5-3 \mathrm{~cm}$.

Flores de hasta $12 \mathrm{~cm}$ long., pericarpelo y tubo con algunas escamas con pelos axilares y a veces una espinita de hasta $5 \mathrm{~mm}$. Tépalos externos verdes, internos blancos, estilo apenas más largo que los tépalos, con estigma de 10-12 lóbulos subulados. Frutos rojo-rubí, pruinosos, de contorno elipsoide a ovoide, de 4-7 cm long., 2-3 cm diám.,

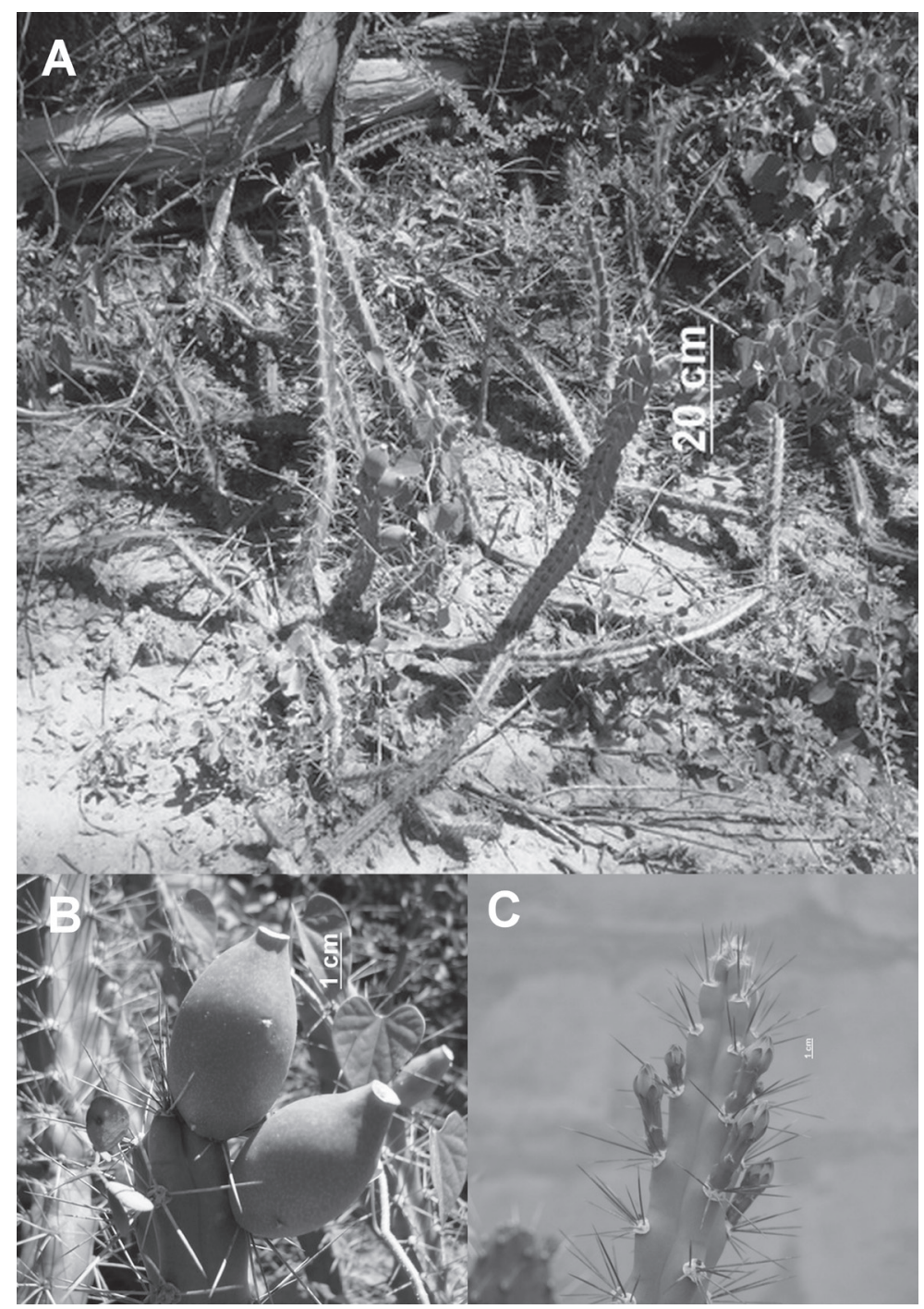

Fig. 1. A: Aspecto general de la planta. B: Frutos. C: Ápice del tallo, con botones florales (Foto: Luciano Galetti). 
lisos, en ocasiones con una o dos escamas pequeñas. Semillas de 1,5-2 mm, negras, brillantes.

\section{Distribución y hábitat. Esta especie crece} solamente en Paraguay y Argentina. En el Chaco paraguayo ha sido coleccionada en el departamento Alto Paraguay y en enclaves chaqueños en el departamento Concepción (Kiesling, 1994; Kiesling et al. 2008). En Argentina, en la provincia de Formosa, donde crece en el sotobosque y borde de bosques xerófilos, formando densas colonias sobre suelos limosos.

Obs. 1: Muy posiblemente Spegazzini (1925: 111) hizo mención a la presencia en Argentina de esta entidad pero bajo el nombre Cereus azureus Parm. ex Pfeiff. (actualmente considerada sinónimo de Cereus aethiops Haw.). Varias evidencias de que en realidad se trataría de $M$. phatnosperma surgen de la descripción que realizó, entre las que se destacan: "costillas son 5 muy obtusas", "semillas lisas", caracteres que no se condicen con los de $C$. aethiops (esta tiene siete costillas como mínimo y las semillas rugosas o verrucosas).

Otra observación interesante que hace Spegazzini (1925) es: "esta especie que parece bastante rara fué en el Cactacearum platensium tentamen, clasificada erróneamente como Cereus Bonplandi Parm pues entonces no había podido conseguir sus flores". Este último taxón -actualmente Harrisia bonplandii (Pfeiff.) Britton \& Rose- posee tallos muy similares a $M$. phatnosperma, por lo que ambas especies pueden ser fácilmente confundidas a campo cuando no presentan flores ni frutos (Kiesling, 1994).

Obs. 2: Kiesling (1994) describió M. kroenleinii, una entidad muy afín a $M$. phatnosperma, con abundantes poblaciones en el chaco seco paraguayo. La validez nomenclatural de esta nueva especie fue cuestionada por Taylor (1995) quien la publica nuevamente pero incluyéndola en el género Cereus, aunque el ejemplar que designó como tipo nomenclatural en realidad se correspondería con lo que Schumann (1899) llamó Cereus phatnospermus, y en ese caso el binomio Cereus kroenleinii N. P. Taylor sería un sinónimo de esta última (Oakley \& Kiesling, com. personal).

Material examinado. ARGENTINA. Prov. Formosa: Dpto. Bermejo, $25 \mathrm{Km}$ al NO de la ruta 32, en cercanías de la Estancia 'La Palizada', $24^{\circ}$ 21' 39,8" S / 60" 49' 50,1" W, 148 m s.m., “al borde de bosque chaqueño", 20/III/2011, Oakley, Mogni, Albute \& Prado 080 (UNR); sin indicación de departamento, Colonia Santa Rosa, 28/XI/1973, Hidalgo s.n. (LIL 540367).

Material adicional examinado. PARAGUAY. Dpto. Alto Paraguay, Estancia 'Guyrá Toro', línea $1,40 \mathrm{Km}$ al N, $19^{\circ} 51^{\prime} 17,1$ ' S / 58 $37^{\circ}$ '21,8” W, "cladodios con 4 ángulos, en frutos", 26/XI/2002, Mereles, Pérez de Molas, Sede \& Eliceche 8947 (FCQ).

\section{Clave para determinar las especies de Monvillea de la Argentina (modificada de Kiesling, 2010)}

1. Tallos de sección circular, con 9-11 costillas. Epidermis verde-oscura. Espinas siempre similares en tallos juveniles y adultos.

2. Plantas arbóreas, con tronco definido y ramas erectas de $3 \mathrm{~cm}$ diám. Flores de ca. $8 \mathrm{~cm}$ long., con tépalos obtusos, redondeados.

M. euchlora

2'. Plantas arbustivas, sin tronco definido, ramas ascendentes o apoyantes hasta de 1-3,5 cm diám. Flores mayores: hasta $12 \mathrm{~cm}$ long., con tépalos acuminados.

3. Tallos ascendentes o apoyantes, de 2-3,5 cm diám., costillas rectas.

M. cavendishii

3'. Tallos rastreros, con tendencia a trepadores (volubles), de 1-1,5 cm diám., costillas rectas o algo espiraladas.

M. krapovickiana

1'. Tallos de sección subcuadrada o pentagonal, con 4-5 costillas. Epidermis glauca o verde-oscura. Espinas a veces disímiles entre tallos juveniles y adultos. 
4. Epidermis glauca, con vetas blanquecinas. Espinas negras, en número de 3/areola y adpresas en tallos juveniles; 4-6/areola y radiantes en los adultos.

M. spegazzinii

4'. Epidermis verde-oscura. Espinas marrones, en número de 6-7/areola, rectas y radiantes tanto en tallos juveniles como adultos.

M. phatnosperma

\section{Discusión y Conclusiones}

Hasta ahora, M. phatnosperma había sido documentada únicamente como endémica del Chaco Paraguayo (Kiesling, 1994; Kiesling et al., 2008). En Argentina se la encontró en el centro-oeste de la provincia de Formosa, donde crece en el sotobosque y en borde de bosques xerófilos dominados por Schinopsis lorentzii (Griseb.) Engl., Aspidosperma quebracho-blanco Schltdl., Ceiba chodatii (Hassl.) Ravenna y Bulnesia sarmientoi Lorentz ex Griseb. De este modo, esta especie continúa circunscripta a los bosques del centro-norte del Gran Chaco (sensu Prado, 1993), y al parecer sería uno más de los taxones endémicos de dicha provincia fitogeográfica, muchos de ellos pertenecientes a la misma familia -v.g. Stetsonia coryne (SalmDyck) Britton \& Rose, Opuntia discolor Britton $\&$ Rose, entre otros. Su registro dentro del Chaco argentino refuerza la entidad de este singular tipo de vegetación xerófila sudamericana.

\section{Agradecimientos}

A las autoridades de los Herbarios de la Facultad de Ciencias Químicas de la Universidad Nacional de Asunción (FCQ) y de la Fundación Miguel Lillo de Tucumán (LIL) por la autorización para examinar ejemplares. A la Universidad Nacional de Rosario (UNR) y al Consejo Nacional de Investigaciones Científicas y Tecnológicas (CONICET) por la provisión de subsidios para la exploración de la vegetación chaqueña de la provincia de Formosa. Al Dr. Roberto Kiesling por sus oportunas sugerencias. Al Agr. Luciano Galetti por el cultivo de parte del material coleccionado.

\section{Bibliografía}

BRITTON, N.L. \& J.N. ROSE. 1920. The Cactaceae 2: 1-241. Carnegie Institution of Washington, USA.

KIESLING, R. 1984. Cactaceae. In: HUNZIKER, A.T. (ed.), Los géneros de Fanerógamas de Argentina. Claves para su identificación. Bol. Soc. Argent. Bot. 23: 85-92.

KIESLING, R. 1994. Monvillea kroenleinii, a new species from Paraguay. Cact. Succ. J. (Los Angeles) 66: 157-165.

KIESLING, R. 2010. Una nueva especie de Monvillea de la Argentina y la validez del género Monvillea (Cactaceae). Bonplandia 19: 59-64.

KIESLING, R., L. FAUNDEZ, J. LAROCCA \& S. ALBESIANO. 2008. Cactaceae. In ZULOAGA, F., O. MORRONE \& M. BELGRANO (eds.), Catálogo de las Plantas Vasculares del Cono Sur (Argentina, sur de Brasil, Chile, Paraguay y Uruguay), pp. 17151830. Monogr. Syst. Bot. Missouri Bot. Gard. 107. Missouri Botanical Garden Press, Saint Louis, USA.

PRADO, D. 1993. What is the Gran Chaco vegetation in South America? II. A redefinition. Contribution to the study of flora and vegetation of the Chaco. VII. Candollea 48: 615-629.

SCHUMANN, K. 1899. Die Cactaceae der Republik Paraguay III. Gattung Cereus. Monatsschr. Kakteenk 9: 164-168.

SPEGAZZINI, C. 1925. Nuevas Notas Cactológicas. Anales Soc. Ci. Argent. 99: 85-146.

TAYLOR, N. P. 1995. Validation of Monvillea kroenleinii Kiesling as Cereus kroenleinii and a note on extension of its range. Kew Bull. 50: 819-820.

Recibido el 16 de agosto de 2012, aceptado el 3 de octubre de 2012. 